\title{
Emergence and dissemination of ecosystem concept in innovation studies: A systematic literature review study
}

\author{
Arash Hajikhani \\ Lappeenranta University of Technology \\ arash.hajikhani@1ut.fi
}

\begin{abstract}
The abundant academic literature on ecosystem approach has attracted the attention of many researchers in various fields (such as business and management, innovation and entrepreneurship). The debate is questioning whether ecosystem as a concept is going towards a matured path. This article, contributes to this debate by applying bibliometric analysis approach on two similar comparable concepts of "system" and "ecosystem" within the boundaries of Business and Management (B\&M) discipline. The co-citation analysis shows that the "system" concept is structured on a stable body of references and disseminated into various fields and subdomains. While the "ecosystem" concept is at early development phase, and it is expected to be identified as a distinguished field which provides explicit added value to innovation related research community. This paper provides the network structure of the collaborating authors, compares the disseminating pattern of the concepts and performs an advance topical analysis of respected literatures.
\end{abstract}

\section{Introduction}

The term ecosystem has been used with a substantial interest by scholars in field of innovation management [1]. The term has been borrowed by variety of scientific domains and indeed has been coopted in the press in the past few years to describe various phenomena $[13,15]$.

Ecosystem is a derived term from system. The difference between ecosystem and system is that an ecosystem is a community of living organisms in conjunction with the nonliving components of their environment (things like air, water and mineral soil), interacting as a system [4]. In simple words, ecosystem is a system formed by an ecological community and its environment that functions as a unit, while system is a collection of organized things; as in a solar system [4].

The ecosystem concept has roots in ecology, where it claims that it consists of the biological community that occurs in some local, and the physical and chemical factors that make up its nonliving or abiotic environment. Ecology is branch which studies living organisms and their interaction with the environment they inhabit [14].

The term has been utilized in fields of business and economics by Rothschild in 1990 [17]. In Rothschild's book "Bionomics", he is promoting the understanding of biology in direction of understanding our economic future. He points to ecological dimensions of economy and elaborates interesting parallel and analogies between business and biology. Bionomics is defined as the branch of ecology that examines the economic relations between organisms (organizations) and their environment [17]. The bionomic perspective illuminates the interaction of forces that maintain stability while spawning changes. Later on, Moore in 1993 [11] took the ground and introduced the term "Business ecosystem" by which he emphasized the essentiality of competition among ecosystem components. The author further stressed the dynamics that regenerate the interactions between organisms and the environment.

Previous works have defined and distinguished the concept of ecosystem and gave it a framework $[6,8,10]$. While, the "ecosystem" phrase itself was not very successful in embedding itself in new literature, it has also been criticized for lack of clarity[13]. The emergence of the concept "ecosystem" in business and management studies, has attracted scholars attention toward tracking this growth and exploring the dissemination of the concept to other fields. The major challenge of any noble and emerging concept is to define itself and disseminate to other disciplines and domains of study. The contribution of this paper is exploring the operationalization ,impact 
assessment of the ecosystem concept and its further dissemination to other disciplines.

One way to analyze the creation of knowledge and its diffusion in an emerging field is to use references co-citation analysis [9,20]. References cocitation analysis is a useful approach when it comes to exploring the knowledge structure of a research domain [21]. This analytical technique also serves to discover knowledge diffusion and influence among a research community. It sheds light on the networks of references, the social construction of a field, and on its intellectual advances. Yet, co-citation analysis does not directly provide insights on future trends. This research has leveraged the advancements in bibliometrics data analytics (refers to statistical analysis of written publications) for exploring the evolutionary path of the concept of ecosystem in B\&M studies.

To contribute to the debate on weather or not the ecosystem concept has been able to establish itself in innovation studies within the boundaries of B\&M discipline, this paper take comparison into the context and decided to take into consideration the "system" concept for benchmarking purposes. The initial investigation showed that both of the concepts (system and ecosystem) have been widely adopted in innovation studies literature, therefore it would be sensible practically and contextually to limit the scope to the innovation studies domain. It will be investigated whether ecosystem has the characteristics of a concept or approach through a bibliometric analysis of the literature within the boundaries of B\&M discipline. On the other spectrum, the concept of system will be investigated to see its usage and adoption overtime since it is a well-established concept and close to the meaning of ecosystem. The comparison of system and ecosystem concept in B\&M discipline will provide a fair comparison ground to observe the dissemination trend of the concepts.

To this end, the literature which adopted the concepts of system and ecosystem within boundaries of B\&M discipline (characteristics such as: major publication venues, main used keywords, influential papers) will be identified. Second, the structures of the literature and the most inflectional scientific articles as the core literature for each of the concepts will be explored. An analysis will be conducted to evaluate the network structure and density of the core literature for each concept. Later on, the analysis will be escalated by encountering the co-citations of the papers which have often cited the core literature and by analyzing their respected domains, the areas which the concepts have been disseminated will be discovered.
The paper is organized as follows; second section presents the methodological approach. Then the bibliometric analysis will be outlined based on the procedure which is described in the methodology section. Thereafter, the findings, discussion and conclusions are presented, the final section.

\section{Methodology and data}

In this section, the method used to identify and analyze the bibliometric data will be presented (consisting of: title, abstract, year of publication, authors, publication venue, keywords, list of references). The searching queries and data collection process will be explained in detail as well.

\subsection{Data collection}

The concept of ecosystem and system which has been adopted in the B\&M discipline will be analyzed. Thereby, the focus is on research articles that addressed the terms or variation of the terms in their titles. For that purpose, Web of Science (previously known as (ISI) Web of Knowledge) has been used as a searching database that includes 90 million documents indexed and is considered to be one of the most important databases for scientific bibliometric data. The Web of Science (WoS) core collection will be incorporated to enrich the coverage to all type of indexed documents.

\subsection{Methodology}

Bibliometric data analysis was conducted as a means to provide quantitative analysis of academic literature [12]. Bibliometrics is known as statistical analysis of written publications and citation analysis. The bibliometric method is based on constructing the citation graph, a network or graph representation of the citations between documents. Many research fields use bibliometric methods to explore the impact of their field, set of researchers, or a particular paper [16].

The bibliometric data analysis was facilitated by the help of toolkit for Network Analysis Interface for Literature Studies "NAILS" that has been developed and published via a conference paper by 2015 [7]. The motivation for using NAILS was to promote its usability and availability as the only open-source cloud based toolkit for bibliometric analysis. Despite of expensive commercialize, closed system tools which are required to be setup and need expert knowledge in data preparation and processing, NAILS proposed an open, extensible tool with even 
more automated workflows which will make this bibliographic analysis available to a wider part of the community of researchers. The literature analysis tool "NAILS", which uses a series of custom statistical and network analysis functions, offers its users an overview of literature datasets retrieved from WoS. (access from: http://nailsproject.net).

The overall process which is conducted for the systematic literature review for the concepts of system and ecosystem is illustrated in Figure 1.

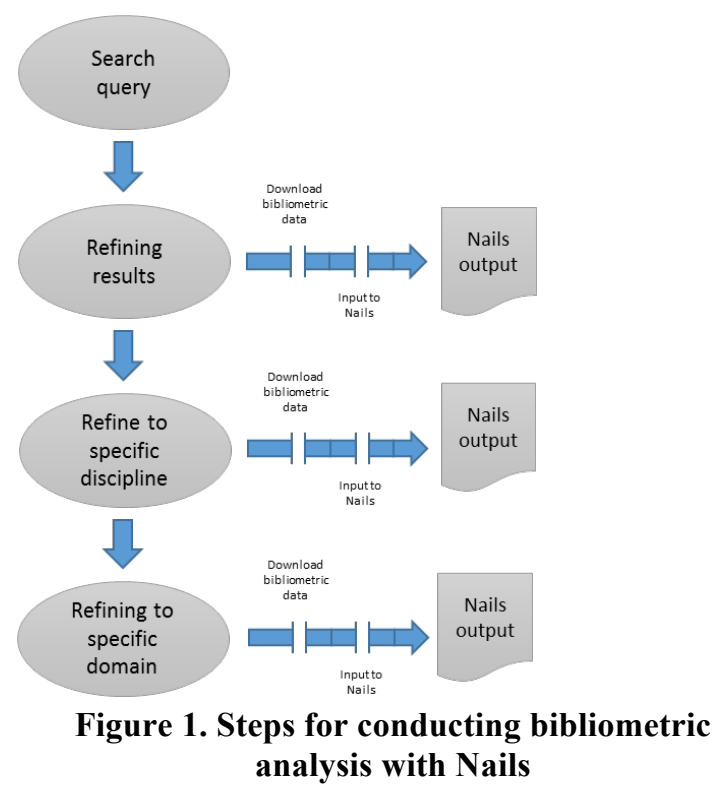

The steps were accordingly:

Step 1: Scientific information retrieved from targeted database (Thomson Reuters Web of Science Core Collection), which was utilized for building the search query with initial keywords. The query was built by using the keywords and boolean operators (e.g. innovate* AND (ecosystem* OR eco-system*)) and then executed.

Step 2: After the query search in the WoS core collection, initial refining (including and excluding of the records) have been done for the result records.

Step 2.1: The refined dataset including the citation data was downloaded in tab-delimited format from WoS manually.

Step 2.2: The downloaded bibliometric data was bundled by a compression tool (option for compression is available in Mac and Windows) and was uploaded onto the Nails (http://hammer.nailsproject.net) online interface.

Once the analysis has been initiated on NAILS, new metrics were calculated such as PageRank (It counts the number and quality of links to a paper to determine a rough estimate of its importance) and In-
Degree (Provides the number of citations coming into a paper in a directed graph) on the citation data of the records. As part of the new metrics, a tailor-made report was generated that provides an abstract/keyword analysis, productive authors/journals and gives recommendations for including top publications based on the citation data. In addition, required data files were also generated in order to graph the network of the records visually.

The Steps 3 and 4 happened as the goal is to dig into a particular domain of study in the concerned discipline. The following sub steps for step 3 and 4 are preceded in the same way as described for the step 2. The bibliometric data from scientific publications were further leveraged for a more extensive and accurate literature analysis. In order to investigate the dissemination of the concerned research domains, the core literature has been detected so to see how often and in what rate the core literature has been cited.

Detecting the "core literatures" is one of the effective ways for distinguishing impactful papers in a concerned domain of study and its relevant literatures [3]. Core literature or documents are advantageous to identify further relevant documents by following the formers' strong and medium-strong links. The notion of core literature was first presented by Small [19] in connection with co-citation analysis. The concept has been escalated by Glänzel and Czerwon [5] on the basis of bibliographic coupling to identify literatures which form important nodes in the network of scholarly communication. In general, the focus on bibliometric analysis is on the citation networks of individual publications. Cooper et al. [3] showed that citation connections could express the relevance to the topic of discussions. Therefore, if a set of records is more highly cited by other publications in a certain domain field, then these records have a greater possibility of belonging to the same domain field.

The interpretation of "core literature" in this paper, represents the most related and impactful papers in the concerned domain of study. Meanwhile, they might not necessarily be interlinked as the concerned discipline might be an emerging one or the topic is highly multidisciplinary in nature. The core literature was then utilized to identify documents which have often cited the core literature. Figure 2 is a good representation of the definition of "core literature" illustrated in this research 


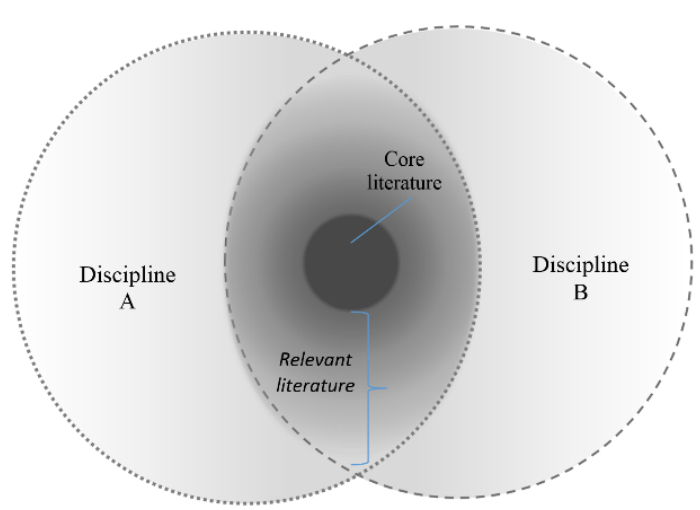

Figure 2. Visualization of the core literature

The process of defining the core literature is manual and the main target is to define the relevancy to the core literature as proxy for filtering the relevant articles. Figure 3 is an illustration of how the practice has been utilized schematically.

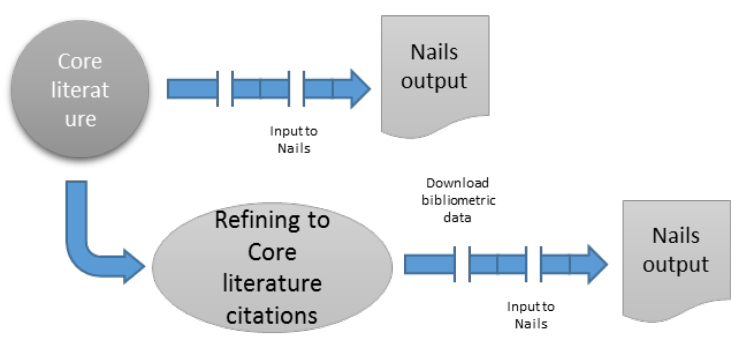

Figure 3. Core literature analysis

After distinguishing the core literature, the records were downloaded and transferred to NAILS for an initial overview (compare with the sub steps 2.1 and 2.2 which were described previously).

The process for collecting the papers which have cited the core literature was manually extensive to conduct, but it is necessary in order to see the dissemination pattern of the core literature. The process includes collecting the full bibliometric data from these references. By retaining the citation's bibliometric data that cited the core literature, it would be clear which papers have cited the most of the core literature and in general to what fields they have been disseminated.

Extracted citations get analyzed within the core literature in NAILS, new indexes were calculated in the NAILS report, which shows an indication of the relevancy of the records in regards to the core literature. The fact that the citations to the core literature depends on availability of them relative to the publication time has been encountered. Therefore, the ratio has been developed that highlights the relevancy of the records to the core literature based on the number of citations which has been made in the papers (Formula 1). "Times cited per year" is another indication which illustrates the quality of the paper based on the average citation which it gets each year.

$$
\begin{aligned}
& \text { Relevance index } \\
& =\frac{\text { Number of Citation to Core Literature }}{\text { Number of Available Core Literature }} \times 100
\end{aligned}
$$

Formula 1. Relevance index

Following section, the applied procedure will be utilized to understand how the system and ecosystem concepts have been adopted in B\&M discipline. A detailed comparison will be constructed for the usage of the two concepts of system and ecosystem within the context of innovation. The detection of the core literature for the two concepts is necessary to understand the dissemination of the concepts into other study domains.

\section{System concept in business and management discipline}

Here, the practice is to show the usage of system approach in B\&M discipline and how innovation studies adopted the concept. The search query was built using the keywords and boolean operators and wildcard like "*" (The use of asterisk $(*)$ as a truncation symbol allowed the databases to look for different endings of the word). The search executed in the title. Usage of system and its variation (i.e. system/s, systematic/s, systemic/s) in WoS core collection for English language has been looked at which the initial results ended up with $1,844,467$ records.

\begin{tabular}{ll}
\hline Search words in Title & system* \\
Time span & August 2016 \\
& All years \\
& WoS core collection: \\
& Indexes: SCI-EXPANDED, \\
& SSCI, A\&HCI, CPCI-S, \\
& CPCI-SSH, BKCI-S, BKCI- \\
& SSH, ESCI, CCR- \\
& EXPANDED, IC. \\
Search refining criteria & English language \\
\hline
\end{tabular}

According to WoS subject categories, more than $50 \%$ of the results are dominated by categories such as electrical engineering, automation, computer science in artificial intelligence, information systems and telecommunications. Refining the initial results into $\mathrm{B} \& \mathrm{M}$ discipline (the domain isolation was 
facilitated by using WoS subject categories) led to 70,083 records.

Due to the big size of the results, only top 3,000 cited publication has been analyzed with the NAILS toolkit in order to have a better overview of the usage of the concept of system in B\&M studies. The keyword analysis indicated; decision support and information systems, supply chain management and simulation as the top popular keywords and user satisfaction, knowledge management and innovation as highly cited keywords (The full report can be found from this link online).

Topic modeling technique has been applied to analyze the abstracts contents. The technique is a type of statistical model for discovering the abstract "topics" that occur in a collection of documents in order to explore hidden semantic structures in a text body [2]. More precisely, the visualized application of the "Latent Dirichlet allocation" introduced by Sievert and colleagues [18] was utilized in order to perform the topic generation of the analyzed abstracts. Figure 4 is an illustration of the popular distant topics/themes related to the system concept used in B\&M discipline. (The interactive visualization for the topical abstract analysis is available from this link online).

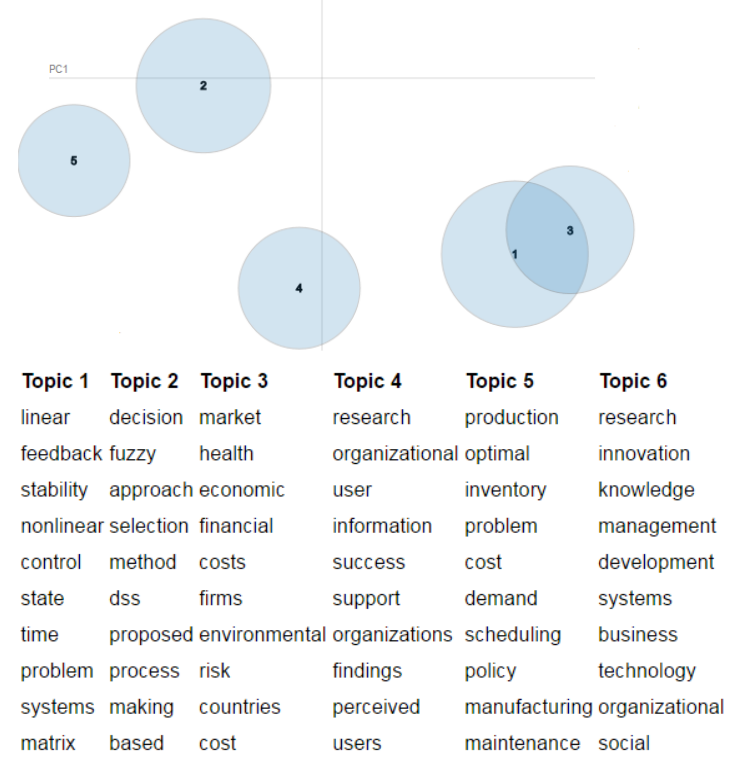

Figure 4. Abstracts topical clustering for system concept in B\&M

The domain boundaries have been narrowed to innovation studies by adding another criterion "innovate*" into the searching query. The results became 2,140 records that had applied the system approach and were within the boundaries of $B \& M$ and contain the context of innovation.

The top popular keywords were: Innovation, regional and national innovation system. Whereas, triple helix (university-industry-government relations) was among the top highly cited keywords after Innovation and innovation system. The top 25 publications have high number of in-degree which shows the interconnectivity of the concept of system in the innovation studies literatures (The full report can be found from this link online).

The process for detecting the core literature was initiated. The 2,140 records were organized and selected according to the PageRank, In-Degree and number of accumulative citation. The title and abstract of the top ranked paper have been read in order to make sure they are within the subject. 67 core literature studies dealing with system concept in business and management with the context of innovation were selected (The NAILS analysis report for core literature from this link online).

The analysis is continued by collecting the bibliometric data of papers which have cited the core literature for the purpose of 1) defining the relevant papers who adopted the concept; 2): understanding the dissemination of the concept into other disciplines. Finally 7,225 full bibliometric data and all the citation of the core literature were extracted (The NAILS analysis online report for the citations to the core from this link online).

Processing the extracted citations analyzed within the core literature in NAILS, Relevance index was calculated in NAILS report, which shows an indication of the relevancy of the records regarding to the core literature. The minimum criteria have been considered for relevancy to core literature to be at least 2 times reference to the core literature. With that criteria, the result ended up to be 1,593 records which have cited the minimum of 2 of the articles in the core literature. To illustrate the dissemination impact, the number of 19,102 citations has been carried out by the 1,593 paper with minimum 2 reference to the core.

Figure 5 shows the distribution of the generated citations based on number of times the papers $(1,593)$ have been citing the core literature. For example, the papers which have 2 of the core literature in their references (a proxy for relevancy), have generated 10,756 citations in total. 


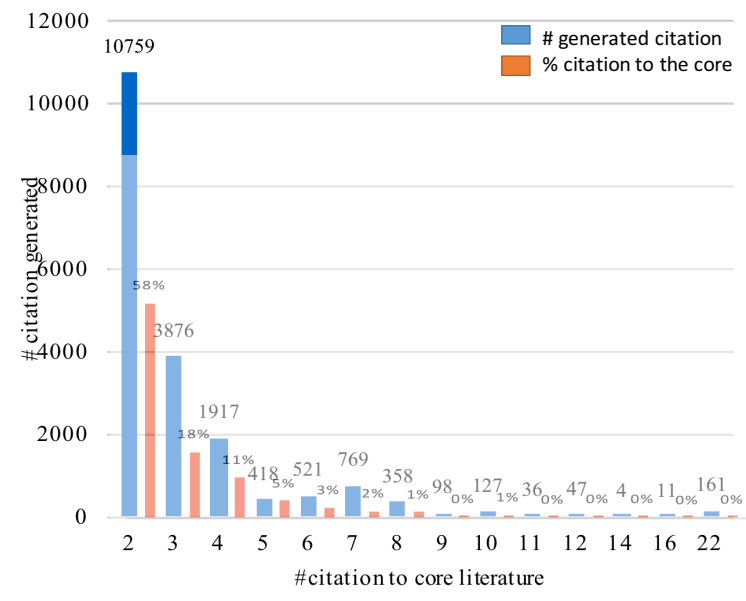

Figure 5 number of citations generated by papers citing the core literature

In Figure 6 an attention has been paid at the subject categories which the papers citing the core literature have managed to penetrate.

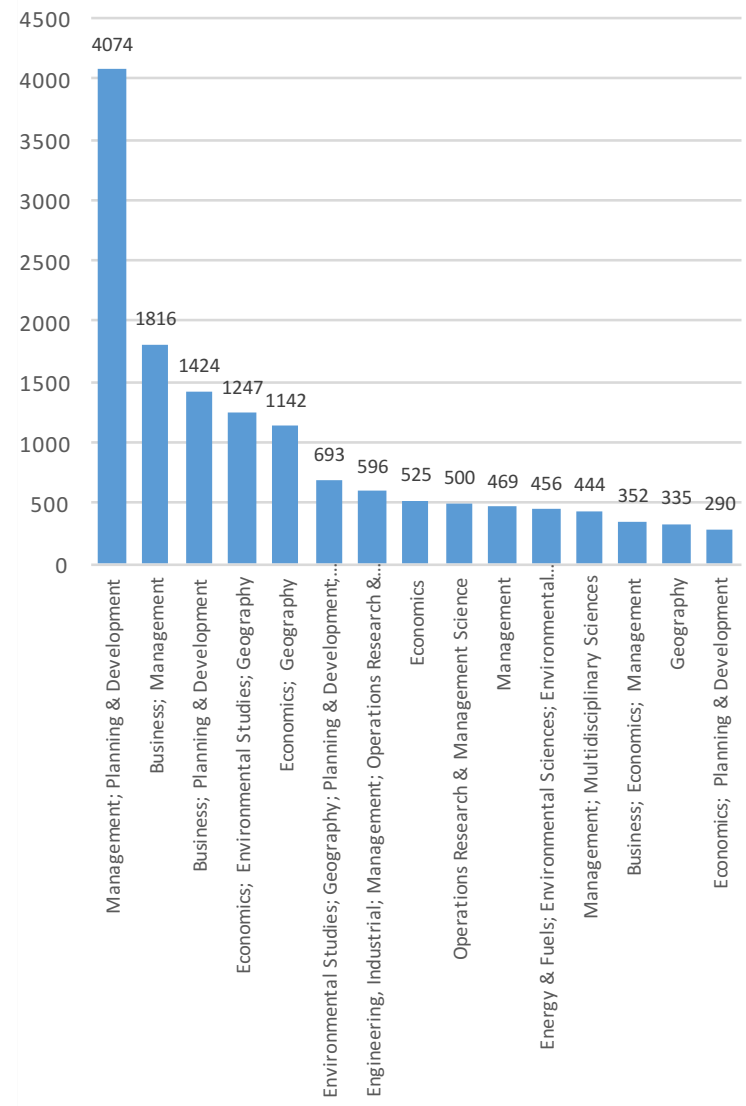

Figure 6. Distribution of the subject category of the core literature citations
As it can be seen from Figure 6, business and economics with various orientations were generating the highest amount of citation and impact, in relative terms. It is also visible that the dissemination of the core concept had an effect in Environmental sciences, Geography and industrial engineering.

\section{Ecosystem concept in business and management discipline}

Looking up the term "ecosystem" and its variations in WoS core collection database, retrieved 38,940 results. A descriptive analysis on the results identified, that the majority use of the term is in ecology, biology, oceanography, forestry and environmental discipline while business and management affiliated materials represents only $6.3 \%$ of the documents. There is much conceptual ambiguity surrounding ecosystems as it had been discussed in introduction. Ecosystems are a metaphor, taken from biology, which is often illdefined. Ecosystem is highly fashionable label therefore its important to notice the underling phenomenon which might be very similar. Other terms have been used extensively to capture ecosystem concept which is needed to be taken into account. Therefore, in order to not to focus on label "ecosystem", the term ecosystem should be expanded. In order to achieve that, terms which associate with the concept of ecosystem are required to be collected. In this regard, a descriptive analysis should be run on the publications which have the ecosystem term in titles in B\&M discipline to see which term associate with ecosystem. The search in WoS for looking up the term ecosystem (i.e. ecosystem*, eco system* and ecosystem*) in WoS core collection database retrieved 643 records. By running a NAILS analysis on the retrieved bibliometric data, it has been noticed that among the popular keywords, terms such as platform, value network, innovation network, quadruple helix and mode 3 innovation ecosystem exists. The keywords were incorporated in the WoS search query.

\begin{tabular}{ll}
\hline $\begin{array}{l}\text { Search words in } \\
\text { Title }\end{array}$ & (ecosystem*) OR ("eco \\
& system*") OR (eco- \\
& system*)OR (platform*) OR \\
& ("value network*") OR \\
& ("quadruple helix") OR \\
& ("innovation network*") OR \\
& ("mode 3 innovation ecosystem*”) \\
Development day & August 2016 \\
Time span & All years \\
Databases & WoS core collection: \\
& Indexes: SCI-EXPANDED, SSCI, \\
& A\&HCI, CPCI-S, CPCI-SSH, \\
\hline
\end{tabular}


BKCI-S, BKCI-SSH, ESCI, CCR

EXPANDED, IC.

Search refining

English language

criteria

The search resulted to 3,260 records in B\&M discipline. The keyword analysis with NAILS indicated popular keywords such as: ecosystem services, innovation, platform, innovation networks and most cited keywords as: ecosystem services and valuation (The NAILS analysis report from this link online).

Figure 7 illustrates the abstract topical cluster analysis for the publication related to ecosystem concept in B\&M discipline boundaries. (The interactive visualization for the topical abstract analysis is available from this link online)
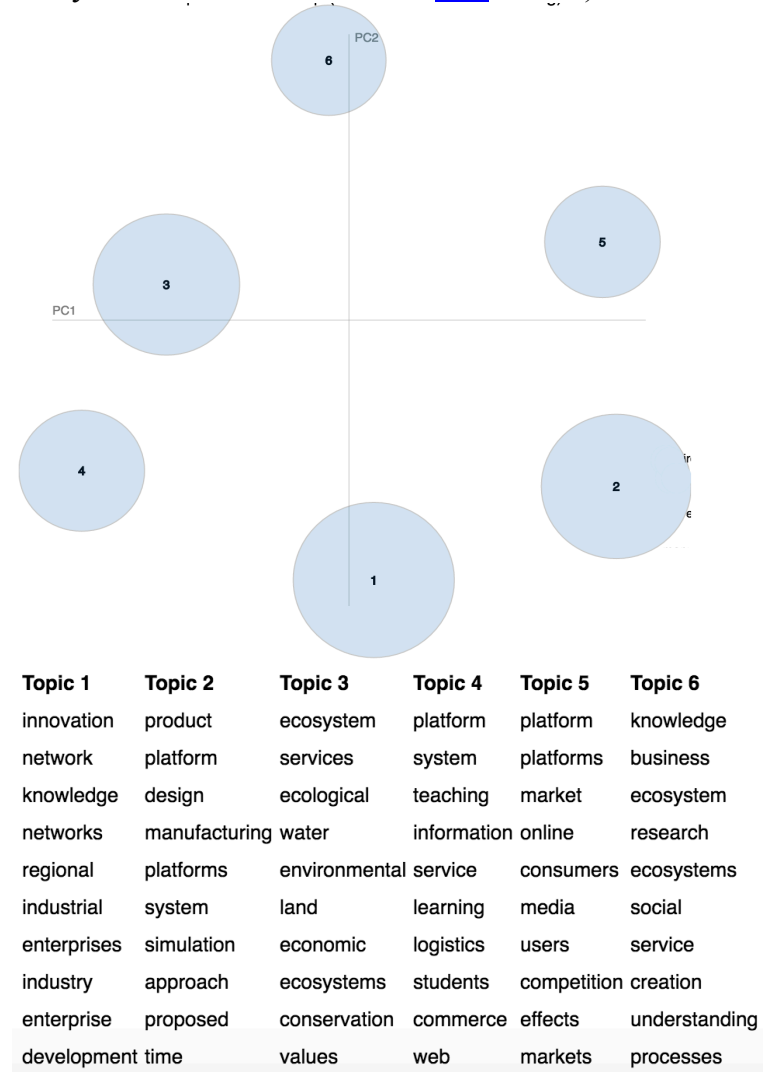

Figure 7. Abstracts topical clustering for ecosystem concept in B\&M

Analyzing the abstracts of the papers with topic modeling (LDA), illustrated that the popular distant topics/themes which ecosystem have been used in $\mathrm{B} \& \mathrm{M}$ discipline. Ecosystem related literature is apparent in different topics $(1,2)$ while platform related literature is apparent in topics $(3,4,5,6)$. Innovation topic is shared with both ecosystem and platform only in topic 1 (which has a healthcare theme).
The study domain boundaries narrowed to innovation studies by adding another criterion "innovat*" into the searching query. The results became 988 which represents the records that have applied ecosystem approach and are within the boundaries of B\&M and contain the context of innovation (The full report can be found from this link online).

The popular keywords were: innovation ecosystem and its combination obviously, open innovation, entrepreneurship, learning processes, collaboration and knowledge management. Whereas the highly cited keywords were: business ecosystem, vertical integration, technological change ecosystem services, social and traditional media, online ecosystems and marketing metrics.

The top 50 publications have relatively much lower In-Degree ratio within the publication that represents the low interconnectivity of literature. For the purpose of detecting the core literature, the 988 records were organized and selected according to the PageRank, In-Degree and number of accumulative citation. The title and abstract of the top ranked paper has been read in order to make sure they are within the subject. 42 core papers dealt with the ecosystem concept in innovation studies within the boundaries of B\&M study (The full report can be found from this link online).

The analysis continues by collecting the papers which have cited the core literature and accordingly their bibliometric data. The purpose for this was to, 1): defining the relevant papers who have adopted the concept, and 2): understanding the dissemination of the concept into other subject categories. 5,335 full bibliometric data were extracted (The full report can be found from this link online). Considering the minimum of 2 references to the core literature, 286 papers have meet the criteria. The distribution of the relevant papers regarding the number of times they have cited the core literature is illustrated in Figure 8.

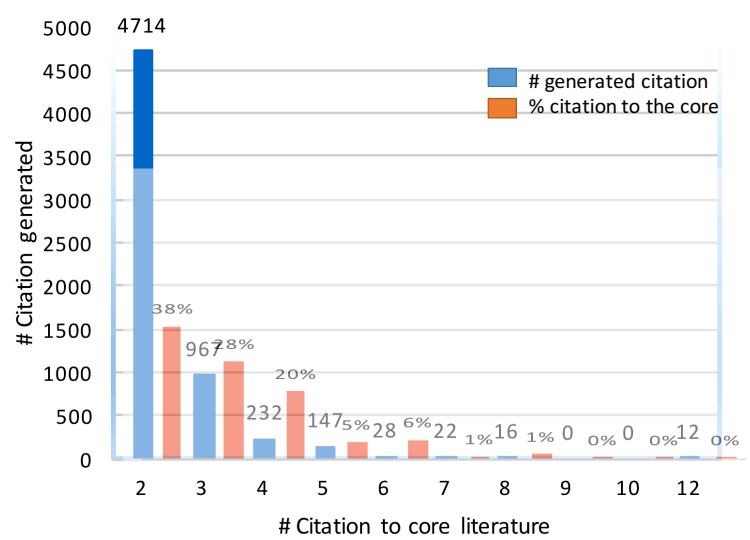

Figure 8. number of citations generated by papers citing the core literature 
In Figure 9, subject categories have been observed so to see the penetration of the core literature.

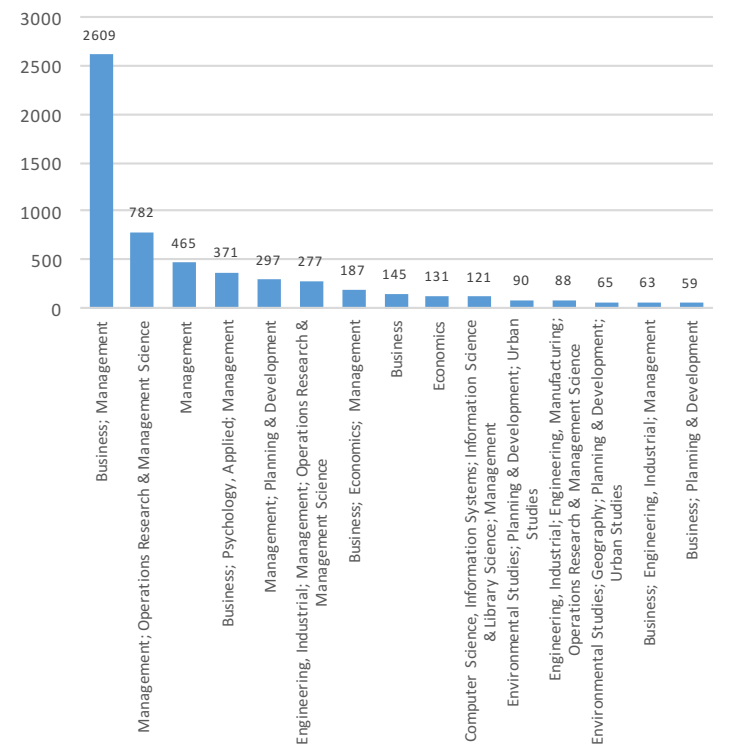

Figure 9. Distribution of the subject category of the core literature citations

The disseminated subject categories which received citation and generate impact are business, management and economy and operation research. The notion of ecosystem within business and management discipline had defused to areas such psychology, computer and information science, environmental, urban and life sciences as well as industrial and engineering.

\section{Findings}

The citation network structure of the two concepts of system and ecosystem in innovation studies within the boundaries of business and management discipline has been reviewed. In the initial phase, the required bibliometric data have been searched and retrieved then based on the defined process the cloud based toolkit for bibliometric analysis "NAILS" had been leveraged to perform the analysis. The investigation was meant to compare the performance and network structure of system and ecosystem concept in innovation studies.

The magnitude of the usage of system concept was 21 times bigger comparing to ecosystem concept in all publication outlets in business and management discipline. Getting to innovation studies, an assessment of the adoption of both concepts revealed that the system concept was only 2 times bigger than the ecosystem concept which is an indication of a relatively bigger contribution of the latter concept into the innovation study domain.

The topical analysis of the abstracts revealed that, papers applying the ecosystem concept are covering more distinct topics than system concept related papers. Comparing the Figures 4 and 7, the six topics clustered papers applying the ecosystem concept have relatively far distance from each other which implies higher diversity in the literature, whereas the topical cluster for the system concept papers have closest distance by each other.

The comparison of dissemination of the two concepts are very informative as it clearly can be seen that the system concept has been disseminated in planning developing and environmental studies in higher extend comparing to ecosystem concept. On the other hand, ecosystem concept was superior in disseminating to areas such as psychology, computer and information sciences and urban studies. The dissemination pattern for the system concept in innovation studies are mainly generated by publications with 2 referred core literature while for ecosystem concept 3 and 4 referred papers has higher portion. The overall dissemination of the system concept in citation terms translated to be 750 papers from core literature which generated over 19,102 citations. The dissemination of the concept of ecosystem to the literature is counted as 286 paper which managed to generate 6,138 citations.

Moreover, an analysis performed on author's network for the both concepts which is shown in the Figures 10 and 11. The network is consisting of nodes which represent authors and edges which represents collaboration or coauthor ship.

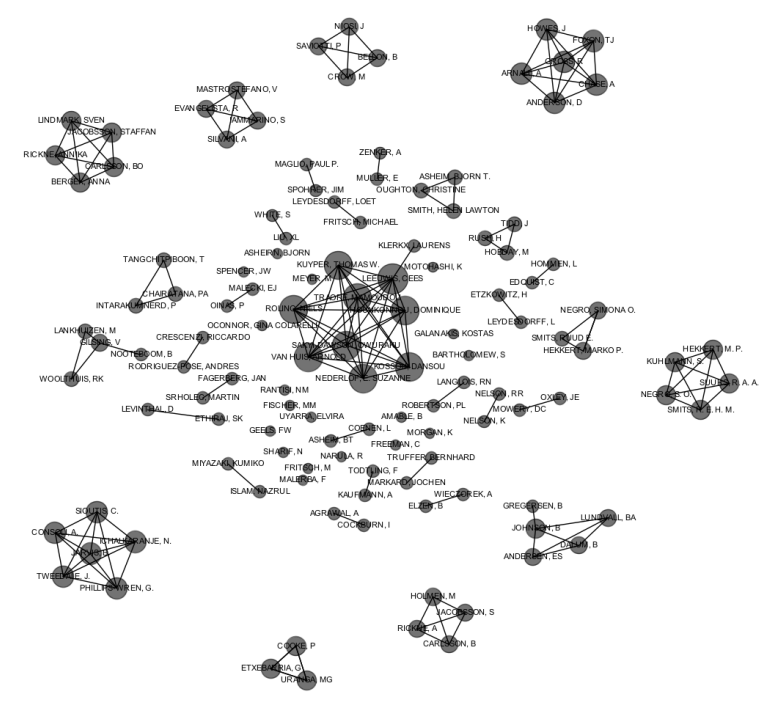

Figure 10. System concept in innovation context 


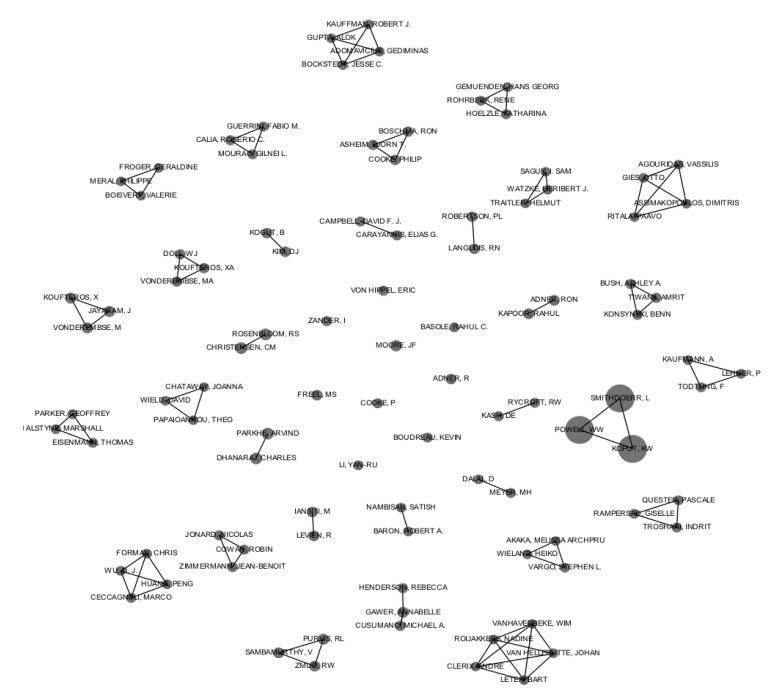

Figure 11. Ecosystem concept in innovation context

Comparing the network structures, it can be observed that less relative collaboration is happening among authors researching the ecosystem concept in the core literature than those focusing on the system concept in the core literature. Translating these patterns to numbers, the average degree (The mean amount of connections per node on the graph) is 2.484 for Figure 10 whereas for Figure 11 equals to 1.835. The other important metric is graph density which is a ratio of the number of edges per node to the number of possible edges. The graph density ratio for Figure 10 is 0.02 while for Figure 11 is lower at number 0.015 . The reported ratio indicates that although there exist highly cited individual nodes in the ecosystem concept core literatures (high InDegree value of nodes), the authors were not able to find each other and collaborate. The result of low level of collaboration for the ecosystem concept leaders is eminent while looking at dissemination pattern of the articles citing the core literature. The diversified and less cited escalated articles are a result of the missing depth and conceptual ground work from the ecosystem concept core literature. It is very dominant that lack of unity in the knowledge base of ecosystem concept in B\&M caused by the variety usage of keywords (e.g. ecosystem, open innovation, entrepreneurship, collaboration, platforms, and networks) which weakens the momentum for the diffusion of the concept.

A further implication from NAILS analysis report of the bibliometric data is that the system concept has focused on communities and venues for publication, while the ecosystem showed a dispersed behavior on appearing in publication venues.

\section{Discussion and conclusion}

This study has contributed to the field of innovation management literature in several ways. First, it introduces a structured approach for analyzing bibliometric data with an orientation of tracking a concept dissemination. This approach offers a new perspective for understanding how a concept or theory has been disseminated and what the patterns of the author's network are. Second, this study offered a methodological approach into an ongoing debate regarding the system vs ecosystem concepts in an innovation studies context, thereby the attempt is to look at the structure of bibliometric data and citation network.

The analysis in this paper presents a deeper understanding of the usage of the concepts of system and ecosystem in business and management as a discipline, by interpreting their bibliometric characteristics, and determining the current maturity of the fields based on their dissemination orientation.

Comparing to a system as a concept, the initiation of the concept of ecosystem in B\&M studies was carried out with a lack of consistency and interconnectivity of authors. One explanation for the fragmentation of author's collaboration might be caused by the new terms usage that eventually removes the connection with older same concept publications. This fact of moving to a new word usage influences the citation in which it departures and loose of origins. This paper suggests that it is important to develop a commonly understood ecosystem vocabulary that allows a comparison among studies. Furthermore, a shortage of micro level case studies to illustrate the usage of the designed frameworks is suggested. A comparison of such case studies looking into innovation system vs innovation ecosystem would help to differentiate the concepts clearly. Further research on innovation and ecosystem would ideally investigate in more detail, what ecosystem concept approach is needed in different situations. The concept evolutionary path should be guided in order to identify theoretical approaches, such as, principles, indices, models, frameworks, and tools. These approaches would form the necessary foundation for future empirical research and theory development and validation.

It is concluded that ecosystem as a newly emerging concept in innovation studies is maturing by the number of publications, but it is still not attached to an epistemological orientation. Considering the maturity of the usage of system in innovation studies within the boundaries of B\&M, this study suggests the use of a unified definition and 
metrics and calls for a collaboration with the authors within the already established community. Concurrently, while the concept ecosystem might add complexity to the current understanding of the system concept that currently dominates $\mathrm{B} \& \mathrm{M}$, the former adds a new perspective or at least pinpoint the aspects which were underestimated previously. it is hoped that this review invites researchers to initiate more rigor research helping to expand our understanding of the concept of innovation ecosystem.

\section{References}

[1] Erkko Autio and D.W.Thomas Llewellyn. 2014. Innovation ecosystems: Implications for innovation management. The Oxford handbook of innovation management, January: 204-228. http://doi.org/10.1093/oxfordhb/9780199694945.013.01 2

[2] David M. Blei. 2012. Probabilistic topic models. Communications of the ACM 55, 4: 77. http://doi.org/10.1145/2133806.2133826

[3] Harris Cooper, Larry Hedges, and Jeffrey Valentine. 2009. The handbook of research synthesis and metaanalysis. Russell Sage Foundation.

[4] MW Dictionary. 2015. Merriam-webster online. Retrieved June 5, 2016 from http://www.merriamwebster.com/dictionary/learning \nhttp://scholar.google.c $\mathrm{om} / \mathrm{scholar}$ ?hl=en\&btnG=Search\&q=intitle:MerriamWebster+Online\#1

[5] Wolfgang Glänzel. 2015. Bibliometrics-aided retrieval: where information retrieval meets scientometrics. Scientometrics 102, 3: 2215-2222. http://doi.org/10.1007/s11192-014-1480-7

[6] DJ Jackson. 2012. What is an Innovation Ecosystem. Arlington, VA: National Science Foundation http://doi.org/10.1017/CBO9781107415324.004

[7] Antti Knutas, Arash Hajikhani, Juho Salminen, Jouni Ikonen, and Jari Porras. 2015. Cloud-based Bibliometric Analysis Service for Systematic Mapping Studies. Proceedings of the 16th International Conference on Computer Systems and Technologies, 184-191. http://doi.org/10.1145/2812428.2812442

[8] Thierry Isckia and Denis Lescop. 2013. Platform- based ecosystems: Leveraging Network- Centric Innovation. November.

[9] Katherine W McCain. 1990. Mapping Authors in Intellecual Space: A Technical Overview. Journal of the American Society for Information Science 41, 6: 433443.

[10] Birol Mercan and Deniz Götkas. 2011. Components of Innovation Ecosystems. International Research Journal of Finance and Economics 76, 76: 102-112. http://doi.org/1450-2887
[11] J. F. Moore. 1993. Predators and prey: a new ecology of competition. Harvard Business Review 71, 3: 75-86. http://doi.org/Article

[12] Jeppe Nicolaisen. 2010. Bibliometrics and Citation Analysis: From the Science Citation Index to Cybermetrics. Journal of the American Society for Information Science and Technology 61, 1: 205-207.

[13] Deog-Seong Oh, Fred Phillips, Sehee Park, and Eunghyun Lee. 2016. Innovation ecosystems: A critical examination. Technovation. http://doi.org/10.1016/j.technovation.2016.02.004

[14] Theo Papaioannou, David V. Wield, and Joanna C. Chataway. 2009. Knowledge ecologies and ecosystems? An empirically grounded reflection on recent developments in innovation systems theory. http://doi.org/10.1068/c0832

[15] Vaida Pilinkienė and Povilas Mačiulis. 2014. Comparison of Different Ecosystem Analogies: The Main Economic Determinants and Levels of Impact. Procedia - Social and Behavioral Sciences 156, April 365-370. http://doi.org/10.1016/j.sbspro.2014.11.204

[16] Dr Alan Pilkington. 2009. Bibliometrics. Royal Holloway. Retrieved from http://personal.rhul.ac.uk/uhtm/001/BibliometricsIndex. $\mathrm{html}$

[17] Michael Rothschild. 1990. Bionomics: Economy As Ecosystem. Retrieved from http://www.amazon.com/Bionomics-EconomyEcosystem-Michael-Rothschild/dp/0805019790

[18] Carson Sievert and Kenneth Shirley. 2014. LDAvis: A method for visualizing and interpreting topics. Proceedings of the Workshop on Interactive Language Learning, Visualization, and Interfaces: 63-70. Retrieved from http://www.aclweb.org/anthology/W/W14/W14-3110

[19] H Small. 1973. Cocitation in Scientific Literature - New Measure of Relationship Between 2 Documents. Journal of the American Society for Information Science 24, 4: 265-269. http://doi.org/10.1002/asi.4630240406

[20] H. Small. 1988. Mapping the dynamics of science and technology. Scientometrics 14, 1-2: 165-168. http://doi.org/10.1007/BF02020250

[21] Howard D. White and Katherine W. McCain. 1998. Visualizing a discipline: An author co-citation analysis of information science, 1972-1995. Journal of the American Society for Information Science 49, 4: 327 355. http://doi.org/10.1002/(SICI)10974571(19980401)49:4<327::AID-ASI4>3.0.CO;2-4 